\title{
WILEY-VCH
}

DOI: 10.1002/ ((please add manuscript number))

Article type: (Full Paper)

\section{A Flexible PMN-PT Ribbon-based Piezoelectric-Pyroelectric Hybrid Generator for Human-Activity Energy Harvesting and Monitoring}

Yan Chen, Yang Zhang, * Feifei Yuan, Fei Ding and Oliver G. Schmidt

Y. Chen, Dr. Y. Zhang*, Prof. F. Ding, Prof. O. G. Schmidt

Institute for Integrative Nanosciences,

IFW Dresden, Helmholtzstraße 20,

01069 Germany

[*] E-mail: yang.zhang@ifw-dresden.de

F. Yuan

Institute for Metallic Materials,

IFW Dresden, Helmholtzstraße 20,

01069 Germany

Prof. F. Ding

Institute für Festkörperphysik,

Leibniz Universität Hannover, Germany

Prof. O. G. Schmidt

Material Systems for Nanoelectronics,

Technische Universität Chemnitz, Germany

Keywords: PMN-PT ribbons, piezoelectric, pyroelectric, flexible, hybrid generator

Abstract:

The rapid advancements of wearable electronics require continued innovation in sustainable power sources and human interactive sensors. An abundance of energy in various forms, such as mechanical, thermal, optical, and sound are ubiquitous in the environment and human activities. Currently, hybrid generators using piezoelectric polymers with relatively low piezoelectric and pyroelectric constants have been fabricated to simultaneously scavenge mechanical and thermal energies. In this work, micro-patterned single-crystal PMN-PT 


\section{WILEY-VCH}

ribbons, which possess excellent piezoelectric and pyroelectric properties, are utilized to build human activities energy harvesting and monitoring systems. The flexible PMN-PT ribbonbased sensor conformally attached on the surface of human skin enables high sensitivity for human body motions and can detect acoustic sounds precisely. The sensor has been used for monitoring temperature-related activities, caused for instance by warm water flow and even light illumination. The multi-functional performance of the PMN-PT ribbon-based hybrid generator shows great potential for self-powered wearable and human activities monitoring devices.

\section{Introduction}

Portable and wearable electronics are of great interest as a result of the growing demands of human interactive applications, which provide the promise of facilitating important advancements in the fields of health monitoring, artificial intelligence, consumer electronics, and humanoid robotics. ${ }^{[1]}$ One of the critical challenges regarding the use of wearable devices lies in the lifetime of the energy sources and periodic replacement or recharging of energy sources. As a promising alternative to nonrenewable external sources, building renewable, portable, and sustainable energy harvesting systems that can scavenge energy from the ambient energy in human activities and surroundings has shown great potential in wearable device applications. ${ }^{[2]}$ There have been plenty of demonstrations of wearable electronics with flexible generators via piezoelectric, pyroelectric or triboelectric transduction mechanisms. ${ }^{[3,}$

${ }^{4]}$ The generators which are conformally in contact with the surface of the human body, can also measure and quantify electrical signals generated by human-activities ${ }^{[5]}$ Recent years, apart from mechanical energy, various other previously ignored energy forms, such as thermal, ${ }^{[6]}$ optical, ${ }^{[7]}$ and sound energy ${ }^{[8]}$ have attracted increasingly more attention. Thermal energy is a ubiquitous and abundant energy source in the environment and our own bodies 


\section{WILEY-VCH}

that could be harvested and serve as a potential source for low-power electronics.

Thermoelectric materials generate electric power from temperature gradients $(\mathrm{d} T / \mathrm{d} x)$ by the Seebeck effect. One of the challenges of thermoelectric materials for energy harvesting is the need of a spatial temperature gradient maintained by additional devices. The pyroelectric effect is an alternative possibility for directly converting heat into electricity. Pyroelectric materials produce electricity from temporal temperature fluctuations $(\mathrm{d} T / \mathrm{d} t)$ which extensively exist due to light, wind, and other heat/cool sources. ${ }^{[9]}$ All pyroelectric materials exhibit an intrinsic piezoelectricity and are naturally regarded as ideal choices for simultaneously harvesting mechanical and thermal energy which are referred to as hybrid generators. Another important characteristic of energy harvesters for wearable electronics is its ability to stretch and flex with body movements without causing any damage. ${ }^{[10]}$ Flexible and stretchable generators can be fabricated using intrinsically stretchable organic materials or appropriate geometrical arrangements of conventional inorganic materials. For example, Poly(vinylidenefluoride) (PVDF) and its copolymers have been fabricated as highly stretchable piezoelectric-pyroelectric hybrid generators. ${ }^{[11]}$ These materials combine structural flexibility, low cost implementation, and ease of processing compared to ceramics. However, the piezoelectric coefficient $d_{33}$ and pyroelectric coefficient $p$ are considerably lower than that of commonly used ceramics. In general, inorganic materials provide exceptional performance compared to currently available intrinsically stretchable materials. ${ }^{[4,12]}$ And yet, due to the brittle nature of inorganic materials, they cannot withstand flexible and stretchable operation modes. Most recently, inorganic materials for flexible generators has been vastly improved upon. Scientists have taken various inorganic materials with ultrathin films or nanowires (NWs) with exhibited good mechanical flexibility to overcome the undesired attributes. Stateof-the-art flexible hybrid piezoelectric and pyroelectric generators often use polymer-mode supporting NWs or thin film piezoelectric materials, like zinc oxide $(\mathrm{ZnO}),{ }^{[13]}$ barium titanite (BTO), ${ }^{[14]}$ and lead zirconate titanate (PZT) ${ }^{[15]}$ etc. Although $\mathrm{ZnO}, \mathrm{KNbO}_{3}, \mathrm{PZT}$, and PVDF 


\section{WILEY-VCH}

represent the potential for developing flexible hybrid generators, considering the power output performance and human activities monitoring sensitivity, the development of new materials is still an active area of research.

Single-crystal (1-x) $\mathrm{Pb}(\mathrm{Mg}, \mathrm{Nb}) \mathrm{O}_{3}-\mathrm{xPbTiO}_{3}(\mathrm{PMN}-\mathrm{PT})$ is a remarkable piezoelectric material with a superior piezoelectric coefficient $\left(d_{33}\right)$ up to $\sim 2500 \mathrm{pC} / \mathrm{N}$. This is almost 4 times higher than that of PZT, 90 times higher than that of $\mathrm{ZnO}$, and 75 times higher than that of PVDF. ${ }^{[16]}$ One approach to enhance thermal energy conversion is to use pyroelectric materials with significantly higher pyroelectric coefficients. The pyroelectric performance of single-crystal PMN-PT also surmount other conventional pyroelectric materials. The pyroelectric coefficient ( $p$ ) of $0.7 \mathrm{~Pb}\left(\mathrm{Mg}_{1 / 3} \mathrm{Nb}_{2 / 3}\right) \mathrm{O}_{3}-0.3 \mathrm{PbTiO}_{3}$ was estimated to be $\sim 1040 \mu \mathrm{C} / \mathrm{m}^{2} \mathrm{~K}$ at room temperature, which is almost 2.7 time higher than that of PZT, 110 times higher than that of $\mathrm{ZnO}$, and 26 times higher than that of PVDF copolymers. ${ }^{[9]} \mathrm{PMN}-\mathrm{PT}$ also possesses low thermal diffusivity $\left(D \sim 4.4 \times 10^{-7} \mathrm{~m}^{2} / \mathrm{s}\right)$, low volume specific heat $\left(C_{\mathrm{v}} \sim 2.5 \times 10^{-6} \mathrm{~J} / \mathrm{m}^{3} \mathrm{~K}\right)$. The piezoelectric-pyroelectric activity of PMN-PT permits concomitant harvesting and measurement of human-activities related mechanical or thermal signals. Emerging techniques in micro-fabrication have extended the application range of PMN-PT to flexible devices. ${ }^{[17]}$ It is perhaps not surprising to develop high-performance flexible hybrid piezoelectricpyroelectric generators and sensors using single-crystal PMN-PT.

In this work, we present a highly flexible hybrid generator/sensor based on ultrathin $(\sim 1 \mu \mathrm{m})$ ribbons of single-crystal PMN-PT, which is capable of laminating on the human skin with capabilities of scavenging mechanical and thermal energy generated by human-activities. By virtue of the outstanding $d_{33}$ and $p$ parameters, simple-structured PMN-PT layers surrounded with SU8 polymer favor higher power output and high sensitivity to small levels of mechanical and thermal disturbances. As proof of concept, we demonstrate that our flexible device can perceive pressure and strain from human motion, as well as from ambient vibrations such as wind and sound waves. Besides, the hybrid generator is also sensitive to 


\section{WILEY-VCH}

surrounding temperature fluctuations induced by warm/cool water and light illumination. All of these functions are highly desirable in self-powered wearable and human activities monitoring devices.

\section{Results and Discussion}

Figure 1a shows a schematic of the fabrication steps. First, a single-crystal 0.7PMN-0.3PT film $(\sim 10 \mu \mathrm{m})$ was obtained from mechanical and chemical polishing of bulk PMN-PT, of which the back side was coated with $\mathrm{Cr} / \mathrm{Au}$. The $10 \mu \mathrm{m}$-thick PMN-PT film was thinned down to $1 \mu \mathrm{m}$ using inductive coupled plasma-reactive ion etching (ICP-RIE). Optical lithography followed by metal deposition was used to pattern the PMN-PT ribbons (Step i). $\mathrm{Ni} / \mathrm{Au} / \mathrm{Ti}$ layers were deposited. The Ni layer worked as the etching mask. After another step of ICP-RIE, the PMN-PT ribbon arrays were fabricated. Then, wet chemical etching was used to release the ribbon arrays from its substrate. After completing the etching of the bottom $\mathrm{Cr}$ adhesive promoter (step ii), Au/Ti/PMN-PT ribbons were laminated to an SU8 stamp and were detached (step iii). Figure 1b shows the scanning electron microscopy (SEM) image. The Au/Ti layers are now at the bottom and will be used as bottom electrode. Opening stripewindows connecting all of them at their ends were fabricated by lithography. Meanwhile, additional windows at the center of each ribbon were also fabricated. Tilted E-beam metal evaporation of $\mathrm{Au} / \mathrm{Cr}$ and following lift-off were then used to fill the opening windows (step iv). All bottom electrodes of each ribbon were then connected, while the deposited metal film in the center served as top electrodes (see Figure 1c). In the next step, we first encapsulated the ribbon arrays into SU8 photoresist. The SU8 here is to separate bottom electrodes from top electrodes. The top electrodes were still left open to air by UV light exposure patterning. Another photolithography and E-beam metal deposition were used to connect all top 


\section{WILEY-VCH}

electrodes (step v, see Figure 1d,1e).The area size of the flexible hybrid generator is around $3 \mathrm{~mm}$ by $3 \mathrm{~mm}$.

The exceptional properties of PMN-PT lie in the optimal composition and orientation of PMN-PT. Compared with bottom-up methods, RIE as a top-down method, can precisely control the composition, orientation during the fabrication and guarantee excellent piezoelectric and pyroelectric performance. X-ray diffraction (XRD) shown in Figure 2a clearly indicates that the PMN-PT ribbons consist of pure-phase, (001)-oriented single-crystal material. Figure 2b shows the energy dispersive X-ray spectroscopy (EDS) of PMN-PT ribbons with indexes of all elements in PMN-PT, which proves the stoichiometry match of PMN-PT ribbons on flexible substrate with that of a single-crystal PMN-PT bulk. As shown in Figure 2c, the current-voltage curve of the as-prepared device shows rectifying behavior reflecting the contributions of Schottky contacts, which also indicates that there is no shortcut between the top and bottom Au electrodes. Figure 2d shows the room temperature polarization-electric field $(P-E)$ hysteresis loop for the PMN-PT ribbons on the flexible substrate recorded at $1 \mathrm{kHz}$. The remnant polarization is around $36.2 \mu \mathrm{C} / \mathrm{cm}^{2}$, which confirms the ferroelectric nature of the PMN-PT ribbons on the flexible substrate. After confirming the high quality of the PMN-PT ribbons, we investigate their performance as a hybrid generator and sensor.

The fabricated flexible PMN-PT ribbon-based device was aimed towards wearable electronics capable of human-activities energy harvesting and monitoring. As shown in Figure 3a, the flexible device was firmly attached to a wrist to detect the strain from the folding and releasing action of the wrist. When the PMN-PT ribbons are under periodic deformation, the charge density on the top and bottom electrodes will be changed. As a result, the current will flow between the bottom and top electrodes. Figure 3a shows that the device produces voltage output up to $1.5 \mathrm{~V}$, when we keep periodic folding/releasing motions of the human wrist. We repeated the folding/unfolding motion for several times, and the generated voltage profiles 


\section{WILEY-VCH}

stayed almost in the same range indicating the detecting stability of the device. Our device can not only detect the large-scale motions, like bending movements of the wrist, but also perceive the subtle motions of the face, neck during speaking and coughing, etc. As shown in Figure 3b, the sensor was put firmly around a human neck, and then the voltage signals were generated when the person was coughing. The sensor precisely captured the signals of the vocal cord vibration. Because the generated electric voltage is perpendicular to the applied strain, the device was working in the $d_{31}$ mode. In order to find out the output performance of the device in the $d_{33}$ mode, we measured the output voltages under external impact force.

Figure 3c shows that the open-circuit voltage of the device reached up to $3.4 \mathrm{~V}$ under a small stick hitting the device at a stimulated frequency of $1 \mathrm{~Hz}$. These results show that the flexible device is capable of human-activity energy harvesting and monitoring.

Sound and music are parts of our everyday sensory experience. To demonstrate the sound detection capabilities, we monitored the piezoelectric response of our sensor to the sound stimuli from a loudspeaker (Figure 4a). Figure 4b shows the piezoelectric voltage waveforms observed in response to a series of alphabet letters such as " $A$ ", "B", "C" and " $D$ " from a loudspeaker. A time-dependent variation of the voltage waveforms of the sensor precisely matches the sound waveforms from the loudspeaker. The result shows that a maximum output voltage of $20 \mathrm{mV}$ is obtained at a sound intensity of $90 \mathrm{~dB}$. The sensor distance from the loudspeaker is kept at $1 \mathrm{~cm}$. On the other hand, the readout voltage signal from a planar PMN-PT film of $10 \mu \mathrm{m}$ thickness does not exhibit any noticeable response under the same detection conditions. Compared to the thick PMN-PT film, owing to the largely reduced size, a relatively small force can induce the mechanical agitation, which is beneficial for building ultrasensitive devices. Our sensor also enables perception of sound waves consisting of music. We played a short clip of the music called 'Tagu' to the sensor over a loudspeaker. The music intensity was kept at $90 \mathrm{~dB}$. As shown in Figure 4c, the timedependent position and intensity of the piezoelectric voltage waveforms and the 


\section{WILEY-VCH}

corresponding spectrograms well agree with the waveforms and spectrograms of the music clip.

Next, in the absence of any strain we measured the pyroelectric output from the device. The device was glued tightly on a hot plate so that no mechanical vibration could happen. The output voltage and current from the pyroelectric effect have been measured by varying the temperature of the hot plate from 295 to $303 \mathrm{~K}(\Delta T \approx 8 \mathrm{~K}, \mathrm{~d} T / \mathrm{dt} \approx 0.2 \mathrm{~K} / \mathrm{s})$, as shown in

Figure 5a. Under forward connection, an output voltage and current pulse peaks were observed up to $0.1 \mathrm{~V}$, and $20 \mathrm{nA}$, respectively, corresponding to a peak power density of 2 $\mathrm{mW} / \mathrm{cm}^{3}$. After a reversal connection to the voltage meter, a reversed output signal was observed (Figure 5b), which confirms the obtained signals in Figure 5a were generated by the pyroelectric effect of the PMN-PT ribbons. If the temperature of the generator from room temperature to a higher temperature, the increase in temperature will result in that the total average spontaneous polarization is decreased. The quantity of induced charges in the electrodes are reduced, resulting in a flow of electrons from external circuit. The pyroelectric current $I$ can be defined as $I=p A(d T / d t)$, where $p$ is the pyroelectric coefficient, $A$ is the electrode area, and $d T / d t$ is the rate of temperature change. From the above results, the obtained pyroelectric coefficient of the PMN-PT ribbon is estimated as $\sim 980 \mu \mathrm{C} / \mathrm{m}^{2} \mathrm{~K}$, which is much larger than that of PVDF and its copolymers. Temperature is one of the most commonly sensed factors in human activities. To illustrate the temperature sensing application, we attached the sensor to a finger, and immersed the finger into warm water $\left(40{ }^{\circ} \mathrm{C}\right)$, as shown in Figure 5c. When the sensor was immersed into the warm water, a negative voltage peak was observed. An opposite output was observed when the sensor was taken out of the warm water and exposed in air. When the sensor undergoes dipping-in and taking-out the water, we didn't observe any symmetric fast response. The sensor was kept in hot water for around 10s to better exclude the piezoelectric effect which happens at short time scale. The positive and negative voltage signals in Figure $5 \mathrm{c}$ are asymmetric, which can be 


\section{WILEY-VCH}

ascribed to the different rate of heat absorption and dissipation during dipping-in hot water and taking-out to air process of the sensor. From the signal profile, we confirm that the pyroelectric effect dominates this process and illustrates the functionality of sensor for temperature detection. The open circuit voltage $(V)$ can be expressed as $V=\left(p / \varepsilon_{33}^{\sigma}\right) h \Delta T$, where $\varepsilon_{33}^{\sigma}$ is the permittivity in the polarization direction at constant stress, $h$ is the material thickness, $\Delta T$ is the temperature change. It can be seen that the pyroelectric sensing potential can be enhanced by increasing the pyroelectric coefficient $p$, the thickness of materials $h$, and the temperature change. Considering flexibility requirements, the thickness of PMN-PT must be largely reduced down to hundreds of nanometers. In human activities monitoring applications, the operation temperature range $(\Delta T)$ is relatively narrow. So the exceptional pyroelectric coefficient $p$ of PMN-PT is of crucial importance for the pyroelectric detection sensitivity.

We have also investigated the pyroelectric response of our device under light illumination. Because the pyroelectric sensing is based on a thermal effect, it is thought to be wavelength independence. If compared to the semiconductor photodetector based on photoelectric effects, free charge carriers are generated when the active material is illuminated by photons of an energy greater than or equal to its bandgap, and thus, the sensitivity of the detector is wavelength dependent. We illuminated the sensor with a $532 \mathrm{~nm}$ laser pointer. The light illumination on PMN-PT causes the temperature to increase. The temperature change induced by light illumination periodically turned on and off results in a maximum output voltage up to $50 \mathrm{mV}$ (Figure 6a). It can be seen that the voltage output peaks increased with increasing the illumination intensity on the device (Figure 6b).

\section{Conclusions}




\section{WILEY-VCH}

In conclusion, we have developed the first inorganic material-based flexible hybrid energy harvesting device. The design of our device involves the fabrication of micro-structures of single-crystal PMN-PT/SU8 composite films, which scavenge the mechanical and thermal energies. For proof-of-concept demonstrations, we have also shown the functionality of human-activities related multimodal stimuli (pressure, strain, sound, temperature, and light illumination) detection. The collective results suggest implementation of human activities sensing and energy harvesting, and provide a solid platform aiming for building self-powered healthy monitoring and interactive sensing systems.

\section{Experimental Section}

Fabrication of the PMN-PT ribbon-based device: The back side of bulk PMN-PT singlecrystal was coated with $\mathrm{Cr}(30 \mathrm{~nm}) / \mathrm{Au}(200 \mathrm{~nm})$. It was glued on Si substrate with epoxy. Then the PMN-PT was thin down to tens of microns by mechanical and chemical polishing. We use ICP-RIE to further thin it down to around $1 \mu \mathrm{m}$. The etching gases are $\mathrm{SF}_{6} / \mathrm{Ar}$ at flow rate $40 / 20 \mathrm{sccm}$. The temperature is set at $0{ }^{\circ} \mathrm{C}$, and $\mathrm{RF}$ power is $300 \mathrm{~W}$ and ICP power is $1500 \mathrm{~W}$ to generate a dense plasma. The etching rate is found to be $190 \mathrm{~nm} / \mathrm{min}$. When the film is thin enough, a slow etching rate was used to fine control the thickness. And this was achieved by reduced the RF power and ICP power to $150 \mathrm{~W}$ and $700 \mathrm{~W}$, respectively. To pattern the film, optical lithography followed by metal deposition were used. The piezo film was deposited with $\mathrm{Ti}(5 \mathrm{~nm}) / \mathrm{Au}(100 \mathrm{~nm})$ as top contact and $\mathrm{Ni}(200 \mathrm{~nm})$ as etching mask. After lift-off, the sample was sent to the RIE chamber and got etched again. The etching was stopped when PMN-PT film was totally etched through. The etching selectivity of Ni and PMN-PT is around 5. Then we release the PMN-PT ribbons from its substrate by removing the 30nm Cr layer by immersing the sample into acid solvent. The PMN-PT ribbons were transferred to flexible substrate by flip-chip bonding. 


\section{WILEY-VCH}

Characterization and electrical measurement: SEM was performed on a Zeiss NVision 40

FIB-SEM . X-ray diffraction (XRD) was carried out using a Bruker D8 Advanced with Co-K $\alpha$ radiation. EDS was performed by a JEOL JSM-6510 scanning electron microscope. The short-circuit and the open-circuit voltage were measured via current and voltage preamplifiers (Standard Research SR570 and SR560), respectively. The electrical voltage generated was analyzed by an oscilloscope (TDS 2024B, Tektronix). A loudspeaker (Logitech Z213) was connected to a mobile phone to generated acoustic sound waves. The intensity of the sound was measured by a $\mathrm{dB}$ meter. A spectrogram of the acoustic waves was displayed by an audio editor program (WaveSurfer). A programmed controllable heater was used to apply a cyclic change in temperature of the devices.

\section{Acknowledgements}

The work was supported by Alexander von Humboldt Foundation, German Research Foundation (DFG) under project DI 2013/2-1, and German Federal Ministry of Education and Research (BMBF) under project Q.Com-H (16KIS0106). The authors thank S. Nestler, M. Bauer, and Dr. S. Harazim for support in fabrication and measurement.

Received: ((will be filled in by the editorial staff))

Revised: ((will be filled in by the editorial staff)) Published online: ((will be filled in by the editorial staff)) 


\section{WILEY-VCH}
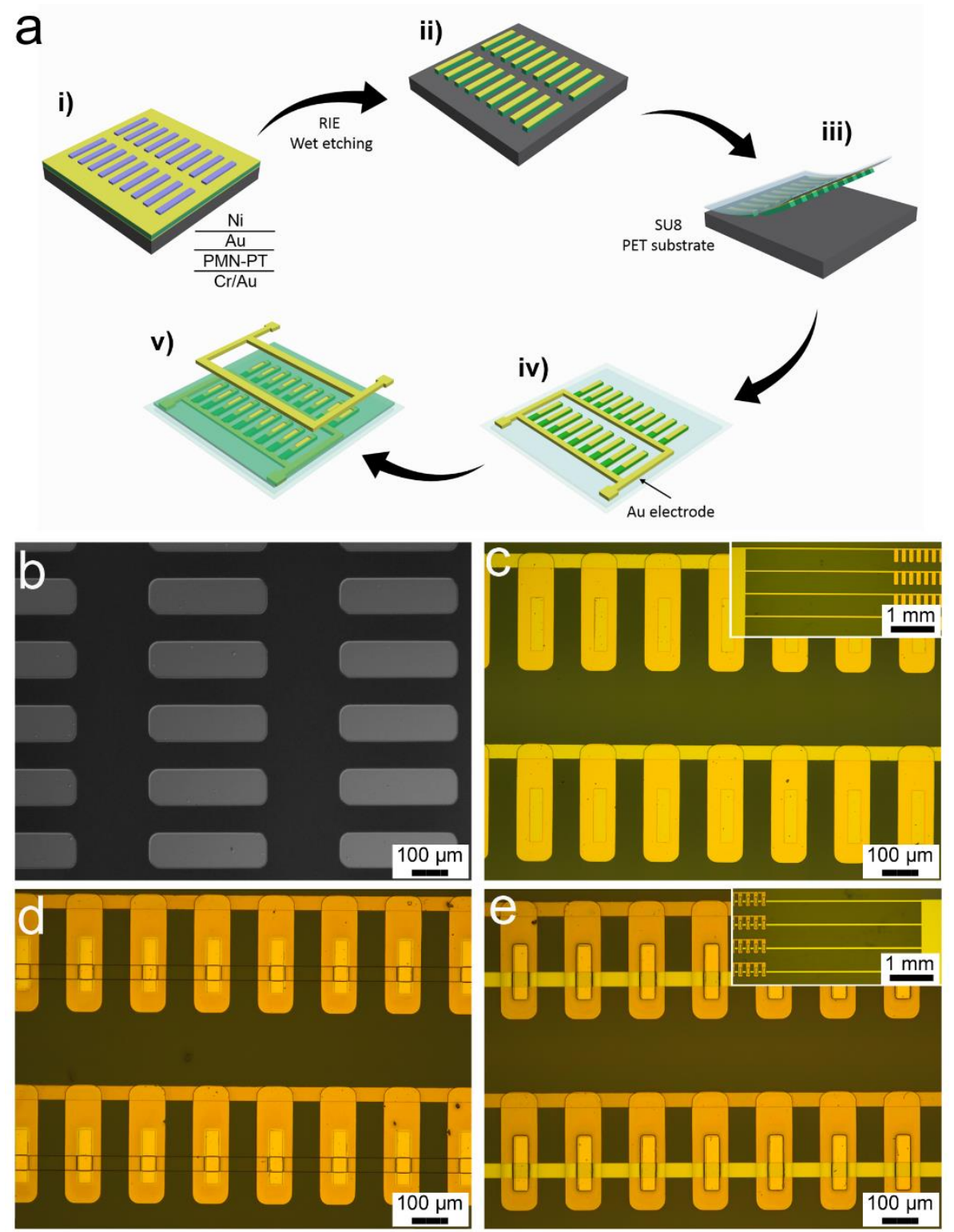

Figure 1. (a) Schematic illustration of the fabrication process for flexible PMN-PT ribbonbased generator and sensor on plastic substrates. (b) A SEM image of the PMN-PT ribbons after RIE etching and transferred to the PET substrate. (c) Optical image of the PMN-PT ribbons with top electrodes and connected bottom electrodes. The inset shows the bottom electrodes were connected and extended for further connection. (d) Optical image of SU8 covered the prepared PMN-PT ribbons. The open windows were used for top electrodes connection. (e) The Au stripes were deposited to connect the top electrodes. The inset shows the top electrodes were connected and extended. 


\section{WILEY-VCH}
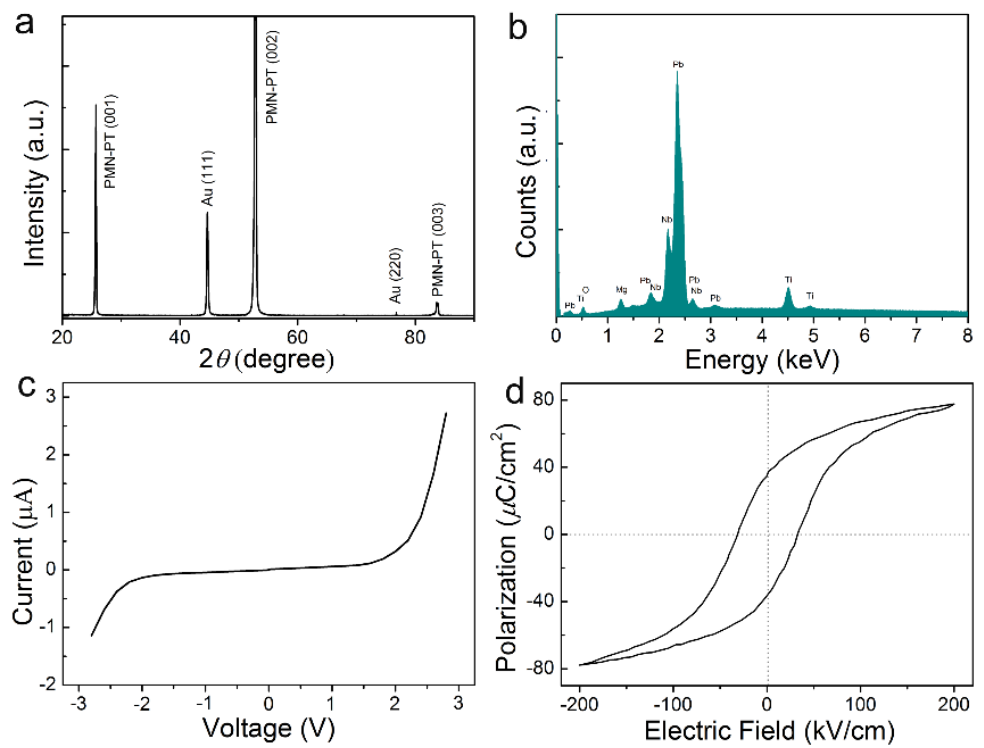

Figure 2. (a) XRD pattern of the PMN-PT ribbon array transferred on PET substrate. (b) EDS spectra of PMN-PT ribbons on PET substrate. (c) Current-voltage curve of the PMN-PT ribbon-based device. (d) Polarization hysteresis of the PMN-PT ribbons on PET substrate.

a
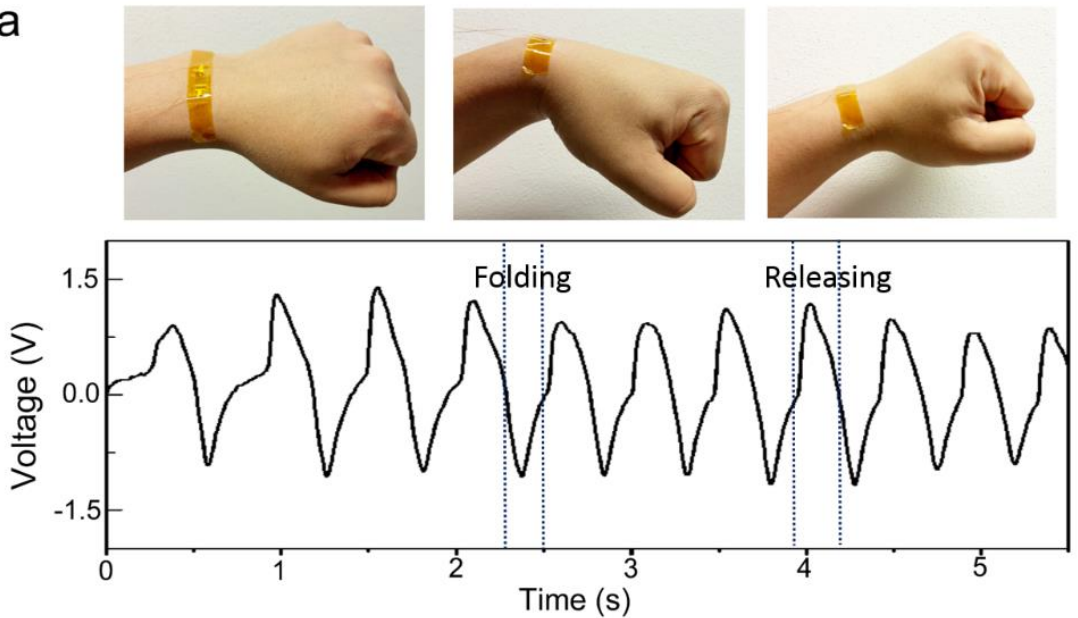

b

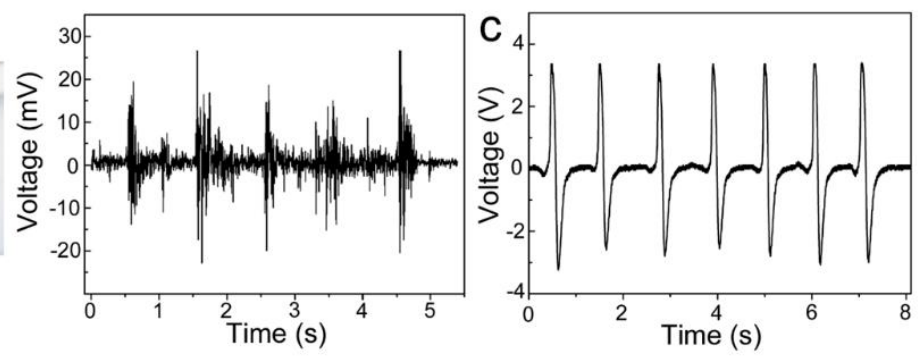

Figure 3. (a) Optical images of the PMN-PT ribbon-based device attached on a wrist. A voltage output appeared on the event of the folding and releasing action of the wrist. (b) A photograph of the device attached on a neck (left). The voltage output waveform for the 


\section{WILEY-VCH}

sensor caused by coughing (right). (c) The voltage output of the device with a small stick knocking.

a

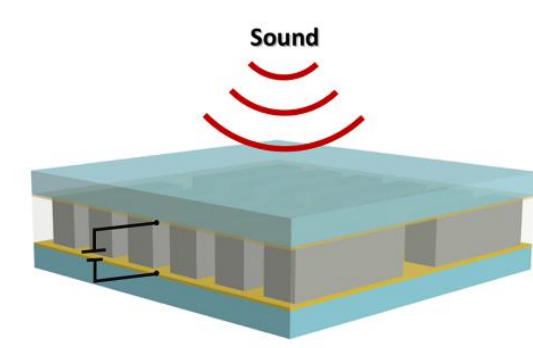

C

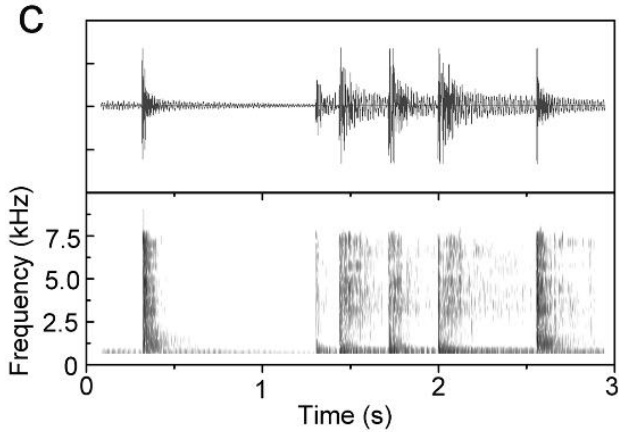

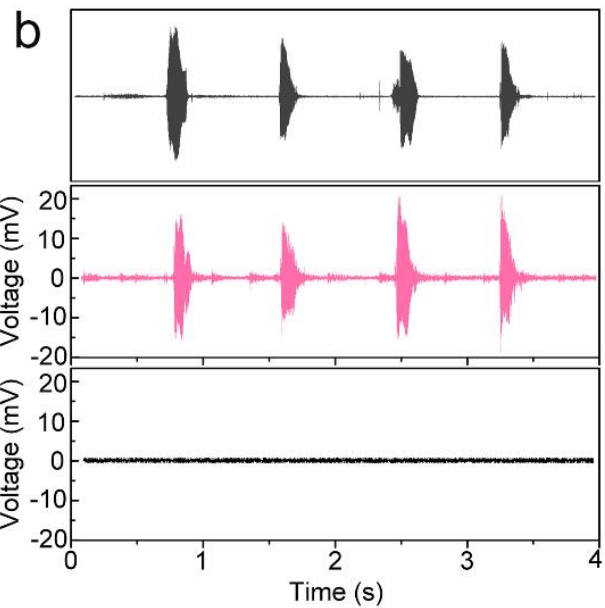

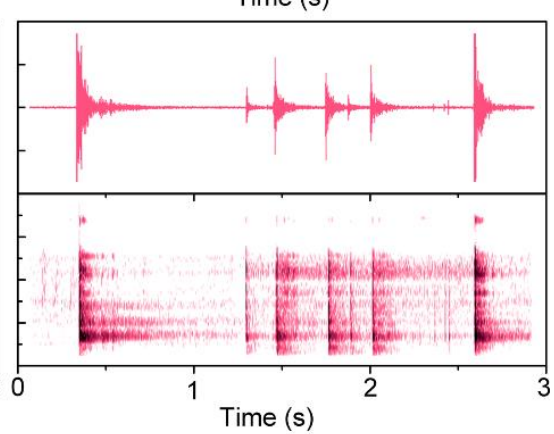

Figure 4. Piezoelectric PMN-PT ribbon-based sensor for acoustic sound detection. (a)

Schematic illustration of the sound detection tests at the sound intensity of $90 \mathrm{~dB}$. The sensor distance from the loudspeaker is kept at $1 \mathrm{~cm}$. (b) The waveform of acoustic sound for different letters of the alphabet (“A”, "B”, “C”, “D”) (top). The voltage output signals from PMN-PT ribbon-based sensor (middle) and planar PMN-PT film of $10 \mu \mathrm{m}$ thickness (down). (c) The waveform and short-time Fourier transform (STFT) signals of the music clip extracted by the sound wave analyzer (black). The readout signals from the piezoelectric sensor. 


\section{WILEY-VCH}
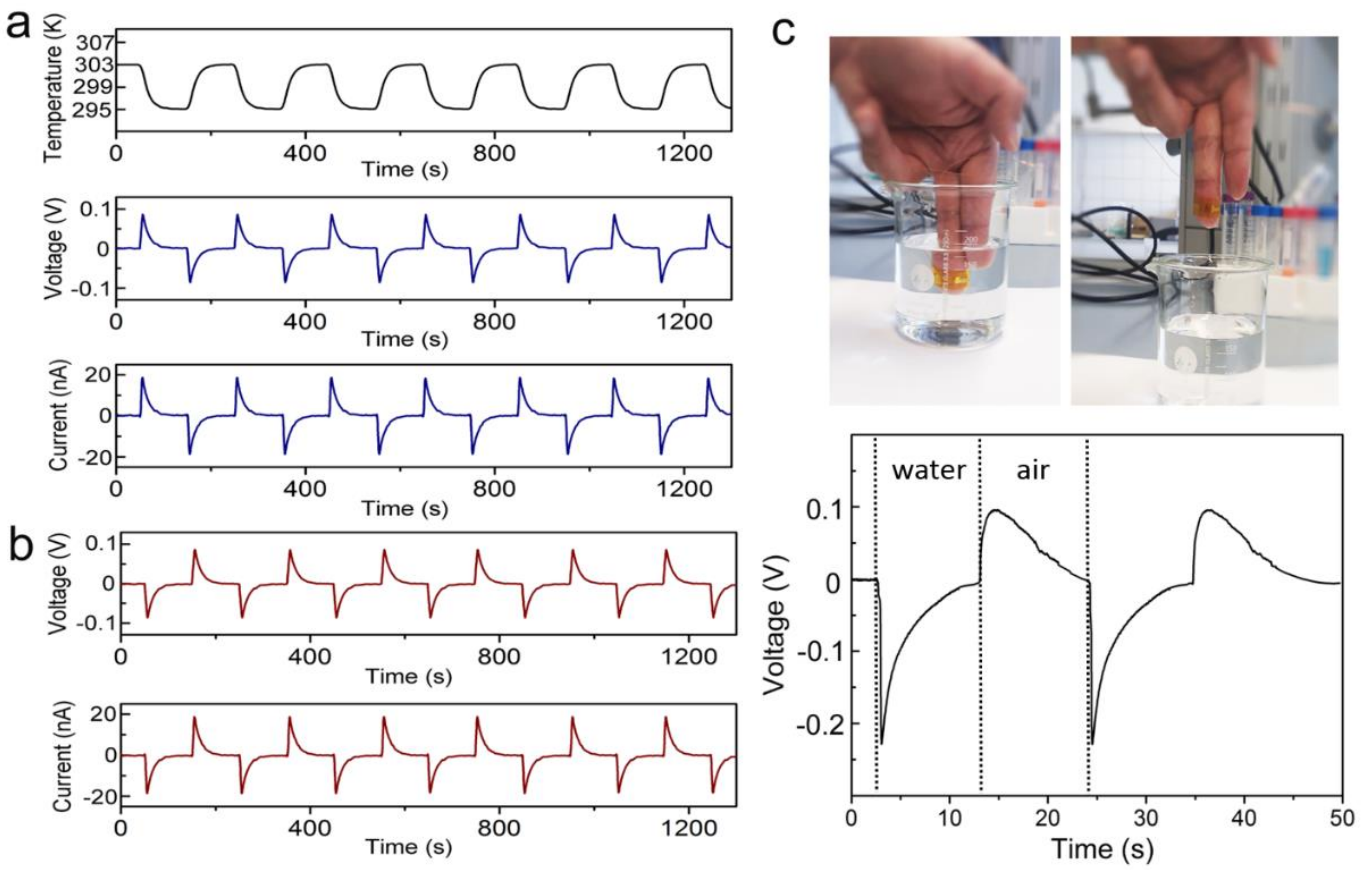

Figure 5. (a) The cyclic changes in temperature of the generator and measured open-circuit voltage and short-circuit current of the PMN-PT ribbon-based pyroelectric generator at forward connection. (b) The measured open-circuit voltage and short-circuit current of the PMN-PT ribbon-based pyroelectric generator at reversed connection. (c) The photographs of the flexible sensor in warm water and air (top). The open-circuit voltage of the pyroelectric sensor under different conditions (bottom).
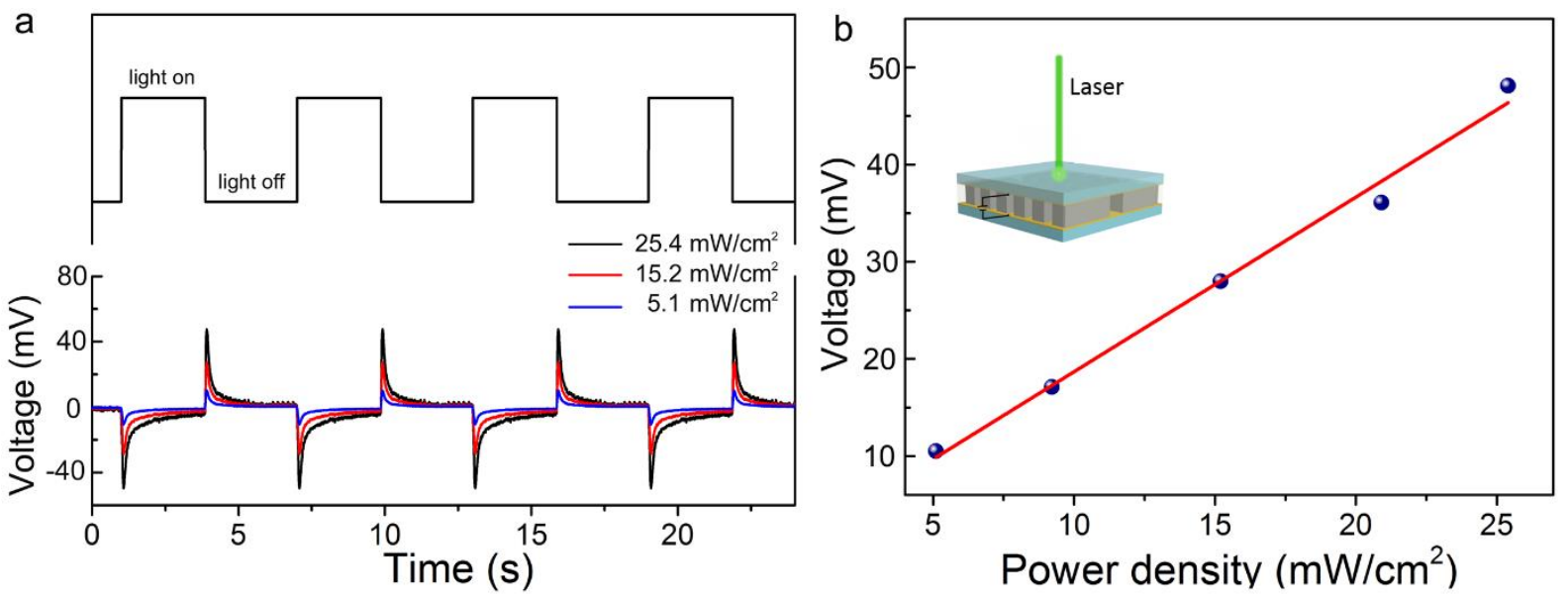

Figure 6. PMN-PT ribbon-based sensor for light detection. (a) The voltage response to on/off light illumination with different power densities for various cycles. (b) The peak output 
WILEY-VCH

voltage of the sensor as a function of the illumination power density. The inset shows the light illumination detection tests. 


\section{WILEY-VCH}

A flexible hybrid generator has been developed using top-down fabricated single-crystal PMN-PT ribbons. The resulting materials offer exceptional piezoelectric and pyroelectric characteristics, to enable high sensitivity for measure and quantify both mechanical and thermal signals generated by human activities. This study indicates great potential applications for portable and wearable electronics.

Keyword: PMN-PT ribbons, piezoelectric, pyroelectric, flexible, hybrid generator

Yan Chen, Yang Zhang,* Feifei Yuan, Fei Ding and Oliver G. Schmidt

\section{A Flexible PMN-PT Ribbon-based Piezoelectric-Pyroelectric Hybrid Generator for Human-Activity Energy Harvesting and Monitoring}

ToC figure ((Please choose one size: $55 \mathrm{~mm}$ broad $\times 50 \mathrm{~mm}$ high or $110 \mathrm{~mm}$ broad $\times 20 \mathrm{~mm}$ high. Please do not use any other dimensions))

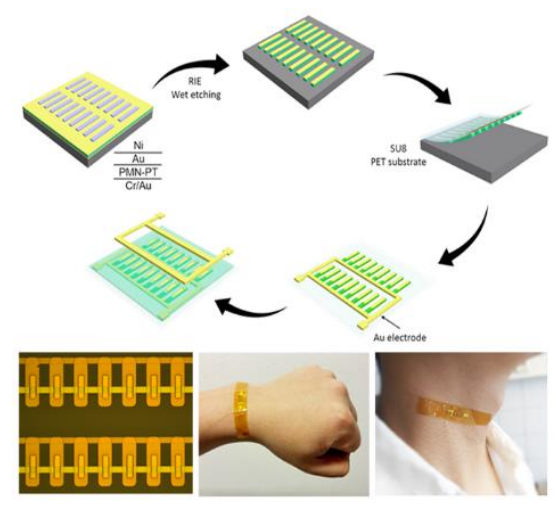




\section{WILEY-VCH}

References

[1] A. Chortos, J. Liu, Z. Bao, Nat Mater 2016, 15, 937; M. Ha, S. Lim, J. Park, D.-S. Um, Y. Lee, H. Ko, Advanced Functional Materials 2015, 25, 2841; N. T. Tien, S. Jeon, D.-I. Kim, T. Q. Trung, M. Jang, B.-U. Hwang, K.-E. Byun, J. Bae, E. Lee, J. B. H. Tok, Z. Bao, N.-E. Lee, J.-J. Park, Advanced Materials 2014, 26, 796; Y. Khan, A. E. Ostfeld, C. M. Lochner, A. Pierre, A. C. Arias, Advanced Materials 2016, 28, 4373; F. Yi, J. Wang, X. Wang, S. Niu, S. Li, Q. Liao, Y. Xu, Z. You, Y. Zhang, Z. L. Wang, ACS Nano 2016, 10, 6519; X. Chen, T. Jiang, Y. Yao, L. Xu, Z. Zhao, Z. L. Wang, Advanced Functional Materials 2016, 26, 4906.

[2] K. Song, J. H. Han, T. Lim, N. Kim, S. Shin, J. Kim, H. Choo, S. Jeong, Y.-C. Kim, Z. L. Wang, J. Lee, Advanced Healthcare Materials 2016, 5, 1572; K. Y. Lee, H.-J. Yoon, T. Jiang, X. Wen, W. Seung, S.-W. Kim, Z. L. Wang, Advanced Energy Materials 2016, 6, 1502566; N. Wu, X. Cheng, Q. Zhong, J. Zhong, W. Li, B. Wang, B. Hu, J. Zhou, Advanced Functional Materials 2015, 25, 4788; S. H. Lee, C. K. Jeong, G.-T. Hwang, K. J. Lee, Nano Energy 2015, 14, 111; T. Q. Trung, N.-E. Lee, Advanced Materials 2016, 28, 4338.

[3] S. Xu, Y. Qin, C. Xu, Y. Wei, R. Yang, Z. L. Wang, Nat Nano 2010, 5, 366; J.-H. Lee, K. Y. Lee, M. K. Gupta, T. Y. Kim, D.-Y. Lee, J. Oh, C. Ryu, W. J. Yoo, C.-Y. Kang, S.-J. Yoon, J.-B. Yoo, S.-W. Kim, Advanced Materials 2014, 26, 765; F.-R. Fan, Z.-Q. Tian, Z. Lin Wang, Nano Energy 2012, 1, 328.

[4] A. Koka, Z. Zhou, H. A. Sodano, Energy \& Environmental Science 2014, 7, 288.

[5] C. Dagdeviren, Y. Su, P. Joe, R. Yona, Y. Liu, Y.-S. Kim, Y. Huang, A. R.

Damadoran, J. Xia, L. W. Martin, Y. Huang, J. A. Rogers, Nature Communications 2014, 5 , 4496.

[6] Y. Yang, W. Guo, K. C. Pradel, G. Zhu, Y. Zhou, Y. Zhang, Y. Hu, L. Lin, Z. L. Wang, Nano Letters 2012, 12, 2833.

[7] Y. Yang, J. H. Jung, B. K. Yun, F. Zhang, K. C. Pradel, W. Guo, Z. L. Wang, Advanced Materials 2012, 24, 5357.

[8] S. N. Cha, J.-S. Seo, S. M. Kim, H. J. Kim, Y. J. Park, S.-W. Kim, J. M. Kim, Advanced Materials 2010, 22, 4726.

[9] C. R. Bowen, J. Taylor, E. LeBoulbar, D. Zabek, A. Chauhan, R. Vaish, Energy \& Environmental Science 2014, 7, 3836.

[10] A. Chortos, Z. Bao, Materials Today 2014, 17, 321; X. Wang, L. Dong, H. Zhang, R. Yu, C. Pan, Z. L. Wang, Advanced Science 2015, 2, 1500169.

[11] J.-H. Lee, H. Ryu, T.-Y. Kim, S.-S. Kwak, H.-J. Yoon, T.-H. Kim, W. Seung, S.-W. Kim, Advanced Energy Materials 2015, 5, 1500704.

[12] G.-T. Hwang, Y. Kim, J.-H. Lee, S. Oh, C. K. Jeong, D. Y. Park, J. Ryu, H. Kwon, S.G. Lee, B. Joung, D. Kim, K. J. Lee, Energy \& Environmental Science 2015, 8, 2677; G.-T.

Hwang, J. Yang, S. H. Yang, H.-Y. Lee, M. Lee, D. Y. Park, J. H. Han, S. J. Lee, C. K. Jeong, J. Kim, K.-I. Park, K. J. Lee, Advanced Energy Materials 2015, 5, 1500051; X. Wang, Nano Energy 2012, 1, 13; J. Kwon, W. Seung, B. K. Sharma, S.-W. Kim, J.-H. Ahn, Energy \& Environmental Science 2012, 5, 8970.

[13] R. Yang, Y. Qin, L. Dai, Z. L. Wang, Nat Nano 2009, 4, 34.

[14] K.-I. Park, S. Xu, Y. Liu, G.-T. Hwang, S.-J. L. Kang, Z. L. Wang, K. J. Lee, Nano Letters 2010, 10, 4939.

[15] X. Chen, S. Xu, N. Yao, Y. Shi, Nano Letters 2010, 10, 2133; K.-I. Park, J. H. Son, G.-T. Hwang, C. K. Jeong, J. Ryu, M. Koo, I. Choi, S. H. Lee, M. Byun, Z. L. Wang, K. J. Lee, Advanced Materials 2014, 26, 2514. 


\section{WILEY-VCH}

[16] G.-T. Hwang, H. Park, J.-H. Lee, S. Oh, K.-I. Park, M. Byun, H. Park, G. Ahn, C. K. Jeong, K. No, H. Kwon, S.-G. Lee, B. Joung, K. J. Lee, Advanced Materials 2014, 26, 4880. [17] S. Xu, Y.-w. Yeh, G. Poirier, M. C. McAlpine, R. A. Register, N. Yao, Nano Letters 2013, 13, 2393; S. Xu, G. Poirier, N. Yao, Nano Energy 2012, 1, 602. 